Reprod. Nutr. Dévelop., 1985, 25 (2), 439-450.

\title{
Régulation de la sécrétion pancréatique par rétroaction négative et hormones gastro-intestinales plasmatiques chez le porc
}

\author{
T. CORRING, J. A. CHAYVIALLE $\left({ }^{*}\right)$, C. SIMOES-NUNES, J. ABELLO
}

avec la collaboration technique de Anne-Marie GUEUGNEAU, Christine BERNARD (*), Georgette BRACHET et F. COINTEPAS

Laboratoire de Physiologie de la Nutrition, I.N.R.A., 78350 Jouy-en-Josas, France.

(*) I.N.S.E.R.M., Unité de Recherches de Physiopathologie, Hôpital E. Herriot, 69834 Lyon Cedex 2, France.

Summary. Regulation of pancreatic secretion in the pig by negative feedback and plasma gastrointestinal hormones.

The aim of this study was to determine the rate of circulating gastrointestinal hormones in pigs when pancreatic secretion was re-introduced or removed from the duodenal lumen.

Permanent fistulas were fitted into the pancreatic duct and duodenum of 16 pigs, and catheters were introduced into the portal vein and a jugular vein as well. Plasma hormone content was determined at different times during two periods of 30 and 60 min each, when the juice was returned to the pigs ; these two periods included a 120-min interval when the juice was not reintroduced into the intestinal lumen.

When the pancreatic juice was not returned to the pigs, plasma secretin content rose significantly in the portal blood $1+42.8 \%$ at $\min 15 ;+52.5 \%$ at $\min 30 ;+31 \%$ at $\min 60$ and $+27 \%$ at $\min 120)$ and in the peripheral blood $(+36.4 \%$ at $\min 30 ;+18.9 \%$ at min 120) compared to the mean values recorded during intraduodenal re-introduction of the secretion. In parallel, the volume of juice secreted and protein output increased significantly but their concentration did not. When the pancreatic juice was removed the mean cholecystokinin level in the portal vein did not change significantly, except at min 30 when its value was $32 \%$ higher than the mean during the periods of restitution. Cholecystokinin level in the peripheral blood was not affected by removing the pancreatic juice from the intestinal lumen. The mean plasma levels of gastrin, somatostatin, VIP and PP in the portal and peripheric blood were unaltered. These results suggest that in pigs secretin may have an important role in the regulation of pancreatic secretion by negative feedback.

\section{Introduction.}

Chez le Rat (Laporte, 1970 ; Green et Lyman, 1972 ; Laporte et Trémolières, 1973), le Porc (Corring, 1974 ; Ihse et Lilja, 1979 ; Vantini et al., 1982) et le Hams- 
ter (Andren-Sandberg et Ihse, 1983), les sécrétions hydroélectrolytique et enzymatique du pancréas exocrine sont soumises à une régulation par rétroaction négative exercée par les enzymes protéolytiques pancréatiques présentes dans la lumière de l'intestin grêle. Une telle régulation n'existerait pas chez le Chien (Sale et al., 1977) et reste controversée chez l'Homme (Ihse, Lilja et Lundquist, 1977 ; Osnes et Hanssen, 1980 ; Dlugosz, Fölsch et Creutzfeldt, 1983). Le mécanisme mis en œuvre dans le phénomène de rétroaction négative n'est pas connu à I'heure actuelle. Une même hypothèse a été avancée par Green et Lyman (1972) et Schneeman et Lyman (1975) chez le Rat, par Corring (1974) chez le Porc et Andren-Sandberg et Ihse (1983) chez le Hamster et concerne le rôle éventuel d'hormones intestinales, sécrétine et cholécystokinine, dans ce mécanisme. Chez le Rat (Green et Lyman, 1972; Schneeman et Lyman, 1975) et le Hamster (Andren-Sandberg et Ihse, 1983), la libération de cholécystokinine, et chez le Porc (Simoes-Nunes, Corring et Chayvialle, 1981) celle de sécrétine et vraisemblablement de cholécystokinine, seraient accrues en l'absence d'enzymes protéolytiques dans la lumière intestinale, ce qui conduirait alors à une stimulation de la sécrétion pancréatique. Cette dernière diminuerait au contraire, comme le taux plasmatique de ces hormones, lorsque les enzymes protéolytiques pancréatiques sont présentes dans l'intestin grêle.

Les résultats d'une étude systématique visant à étudier les taux circulants de quelques hormones gastrointestinales lorsque le suc pancréatique est, soit réintroduit, soit dérivé de la lumière duodénale chez le porc, sont rapportés ici. Cette étude a porté non seulement sur les taux plasmatiques de la sécrétine et de la cholécystokinine mais également sur ceux de la gastrine, de la somatostatine, du VIP (vasoactive intestinal peptide) et du PP (polypeptide pancréatique), régulateurs possibles de la sécrétion du pancréas exocrine.

\section{Matériel et méthodes.}

Animaux. - Vingt-cinq porcs de race Large White, de poids vif moyen $45 \pm 5 \mathrm{~kg}$ ont été utilisés. Ils ont été munis de fistules permanentes du pancréas exocrine et du duodénum selon la technique décrite antérieurement (Corring, Aumaitre et Rérat, 1972) et de deux cathéters placés l'un dans la veine porte en aval du confluent avec la veine pancréatico-duodénale et l'autre dans une veine jugulaire (sang périphérique).

Les animaux ont reçu un régime de croissance standard contenant $16 \%$ de protéines, distribué à $9 \mathrm{~h} 00$ et $17 \mathrm{~h} 00$ en deux repas constitués chacun de $800 \mathrm{~g}$ de farine diluée dans $1600 \mathrm{ml}$ d'eau.

Schéma expérimental. - L'expérimentation a débuté pour tous les animaux 8 jours après l'intervention chirurgicale. Au cours de cette réhabilitation postopératoire, le suc pancréatique était recueilli en permanence dans un flacon maintenu dans de la glace et réintroduit par la fistule duodénale à l'aide d'une pompe péristaltique à débit variable (Corring, Aumaitre et Rérat, 1972).

L'expérimentation, conduite sur l'animal à jeun (le dernier repas est distribué 16 heures avant la période expérimentale), a consisté à répéter sur tous les ani- 
maux l'essai suivant : 1re période au cours de laquelle le suc pancréatique continuait d'être restitué à l'animal $(30 \mathrm{~min}), 2^{e}$ période au cours de laquelle le suc était dérivé de la lumière intestinale $(120 \mathrm{~min}), 3^{e}$ période au cours de laquelle le suc était de nouveau restitué au niveau duodénal $(60 \mathrm{~min})$. Deux à trois essais ont été réalisés sur le même animal. Pendant la période de dérivation de la sécrétion pancréatique, une solution saline de $\mathrm{pH} 8,4\left(\mathrm{NaCl}: 0,6 \%, \mathrm{NaHCO}_{3}: 1,2 \%\right)$ était infusée au niveau duodénal avec une pompe péristaltique au débit de $100 \mathrm{ml} / \mathrm{h}$ pour l'ensemble des essais (Corring, 1974).

Un échantillon de 2 à $3 \mathrm{ml}$ de suc pancréatique était prélevé toutes les $30 \mathrm{~min}$ après le début de l'essai et conservé à $-30^{\circ} \mathrm{C}$ avant analyse. Les prélèvements de sang portal et périphérique ont été réalisés aux temps suivants : en début et fin de la $1^{\text {re }}$ période, toutes les $15 \mathrm{~min}$ au cours de la première heure de dérivation du suc, 90 et $120 \mathrm{~min}$ après le début de celle-ci ; 15, 30 et $60 \mathrm{~min}$ au cours de la $3^{e}$ période. Le sang était prélevé dans des tubes placés dans de la glace et contenant $10 \mathrm{U}$ d'héparine et $500 \mathrm{UKI}$ de Trasylol $\mathrm{R}$ par $\mathrm{ml}$ de sang. Le sang a été centrifugé immédiatement après prélèvement et le plasma conservé à $-30^{\circ} \mathrm{C}$ avant analyse.

Chaque essai sur le même animal était séparé du suivant de 48 heures au minimum.

Analyses. - Le volume de suc pancréatique écoulé a été mesuré toutes les 30 min et les protéines totales déterminées dans chacun des échantillons prélevés (Lowry et al., 1951). Sur chaque échantillon de plasma, on a effectué les dosages radio-immunologiques de la sécrétine, la cholécystokinine, la gastrine, la somatostatine, le VIP et le PP.

La gastrine, la sécrétine, la somatostatine et le VIP ont été mesurés selon des techniques précédemment rapportées (Pelletier, Chayvialle et Minaire, 1978 ; Chayvialle et al., 1978, 1980). Le PP a été dosé selon une méthode dérivée de celle de Schwartz et al. (1976) avec des réactifs donnés par Chance. Le PP était utilisé comme standard et pour marquage à l'iode-125 (IMS 30, Amersham) avec la lactoperoxydase. Le traceur a été purifié par chromatographie liquide à haute performance en phase inverse $($ Bondapak C18, triethyl ammonium phosphate $\mathrm{pH} \mathrm{3,5/acetonitrile,} \mathrm{68/32).} \mathrm{La} \mathrm{variation} \mathrm{intra-dosage} \mathrm{était} \mathrm{de} \mathrm{7,3 \% ,} \mathrm{la} \mathrm{variation}$ inter-dosages de 9,1\%. La concentration plasmatique de cholécystokinine a été mesurée avec un antisérum de lapin $(67 \mathrm{H})$ qui reconnaît à équivalence CCK 33 , CCK 39 et les formes moléculaires carboxy-terminales sulfatées de 9 amino acides ou plus. La réactivité de l'octapeptide C-terminal sulfaté était de $30 \%$, celle du peptide désulfaté et celle de la gastrine étant inférieures à $3 \%$. Le traceur était préparé à partir du décapeptide $\mathrm{C}$-terminal sulfaté (don du $\operatorname{Pr}$. E. Wünsch), marqué par le réactif de Bolton et Hunter mono-iodé (NEN). La purification a été réalisée selon Fourmy et al. (1982). Les plasmas ont été testés contre une courbe standard préparée avec du plasma provenant de chaque animal, et épuré sur charbon Norit A. La récupération de la CCK 33 et celle de la CCK 8 ont varié entre 62 et $80 \%$. La variation intra-dosage était de $9 \%$, la variation inter-dosages de $13,5 \%$. 
Expression des résultats. - Afin de réduire au maximum les variations individuelles qui atteignent fréquemment 40 à $50 \%$ chez les animaux fistulés dans les études sur la physiologie digestive, les valeurs obtenues lors de la dérivation du suc pancréatique ont été exprimées en pourcentage de la valeur moyenne des données correspondantes déterminées pendant les deux périodes de réintroduction du suc pancréatique dans la lumière duodénale. Ce mode d'expression a été appliqué à l'intérieur de chaque essai défini ci-dessus et le résultat rapporté correspond à la moyenne des pourcentages provenant de l'ensemble des essais. Toutes les données ont été exploitées en utilisant le test $t$ de Student non apparié.

\section{Résultats.}

Sur les 25 porcs opérés, 9 animaux ont été éliminés de l'étude à la suite d'un écoulement défectueux du suc pancréatique ou d'une obstruction des cathéters placés dans la veine jugulaire ou porte et 36 essais ont été réalisés au total sur 16 porcs utilisés.

Quantité de protéines totales sécrétées (fig. 1).

Dans les 30 min qui ont suivi la dérivation du suc, la quantité de protéines totales sécrétées par le pancréas exocrine a augmenté significativement $(P<0,01)$ et atteint au bout de 60 min près de $280 \%$ de la valeur moyenne cal-

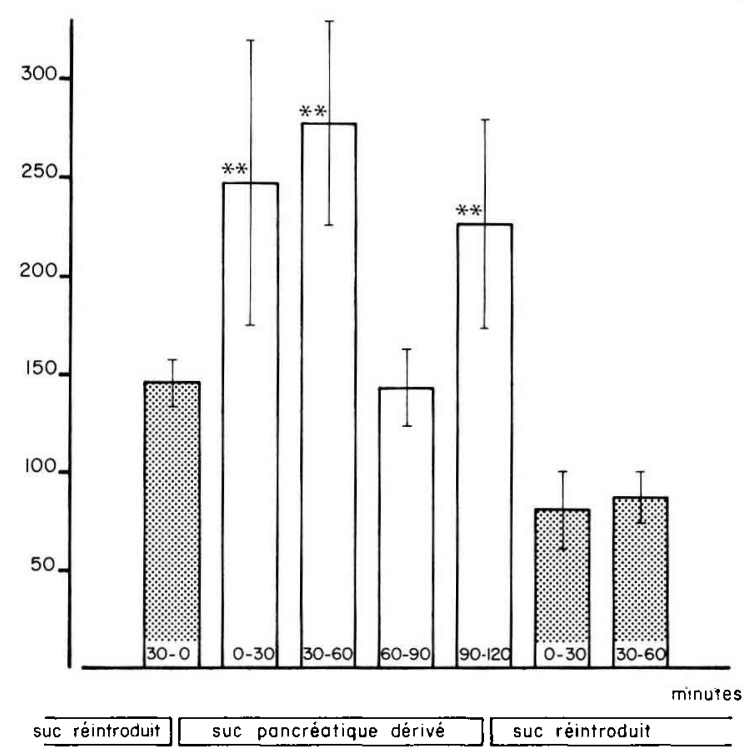

FIG. 1. - Quantité de protéines totales sécrétées par $30 \mathrm{~min}$ par le pancréas exocrine du porc pendant la période de dérivation du suc de la lumière intestinale. Valeurs exprimées en pourcentage de la moyenne des données déterminées pendant les 2 périodes de restitution du suc à l'animal. ** $P<0,01$. 
culée pendant l'ensemble des périodes de restitution de la sécrétion $(151,5 \pm 7 \mathrm{mg} / 30 \mathrm{~min})$. Après une forte diminution des valeurs entre la $60^{\mathrm{e}}$ et la $90^{\mathrm{e}} \mathrm{min}$, on a constaté une nouvelle augmentation significative $(\mathrm{P}<0,01)$ de cette quantité $(227 \%)$ dans les 30 dernières min de la période de dérivation. La restitution de la sécrétion pancréatique à l'animal entraîne un effondrement immédiat des valeurs $(80,5$ et $87 \%$ de la valeur moyenne déterminée sur l'ensemble des périodes de restitution du suc pancréatique).

Volume du suc pancréatique écoulé (fig. 2).

La dérivation du suc pancréatique s'est traduite dès les premières $30 \mathrm{~min}$ et au cours de la première heure par une augmentation significative $(P<0,01)$ du volume de la sécrétion (près de $290 \%$ de la valeur moyenne obtenue pendant les périodes de restitution du suc, soit $37,4 \pm 4 \mathrm{ml} / 30 \mathrm{~min}$ ). On a constaté une nette diminution des valeurs entre la $60^{\mathrm{e}}$ et la $90^{\mathrm{e}} \mathrm{min}$ et une nouvelle augmentation significative $(306 \%, \mathrm{P}<0,01)$ de la $90^{\mathrm{e}}$ à la $120^{\circ}$ min de la période de dérivation. La restitution du suc à l'animal a immédiatement entraîné un effondrement des valeurs (69 et $86 \%$ de la valeur moyenne déterminée sur l'ensemble des périodes de restitution du suc pancréatique).

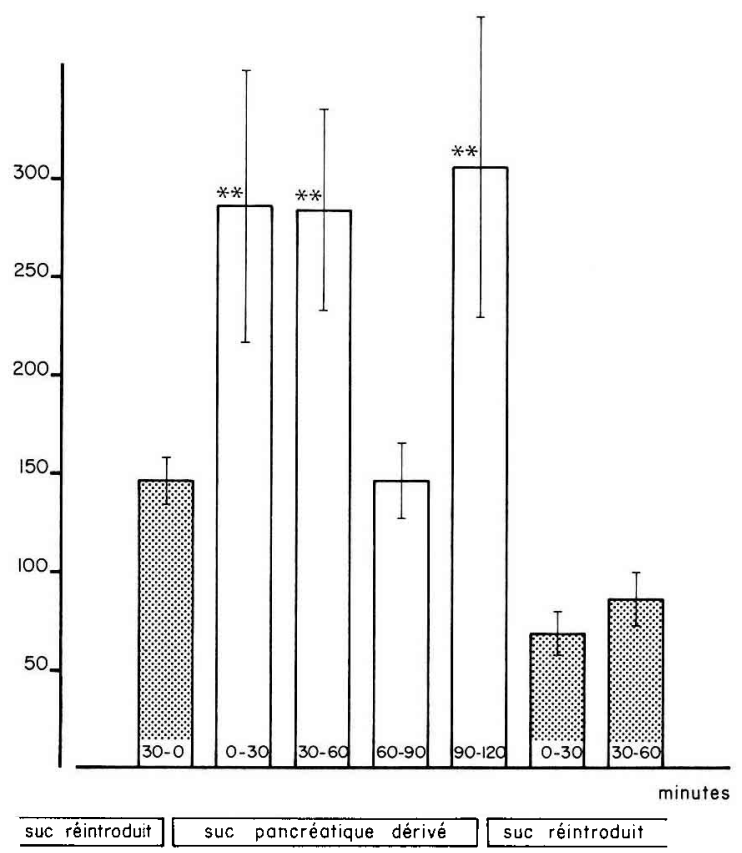

FIG. 2. - Volume de suc pancréatique sécrété par 30 min chez le Porc pendant la période de dérivation du suc de la lumière intestinale. Valeurs exprimées en pourcentage de la moyenne des données enregistrées pendant les 2 périodes de restitution du suc à l'animal. ${ }^{*} P<0,01$. 
Concentration du suc pancréatique en protéines (fig. 3).

On constate sur la figure 3 que la dérivation du suc pancréatique n'a entraîné aucune variation significative de la concentration en protéines totales de la sécrétion du pancréas exocrine. Seule une tendance à la diminution a été observée au cours de la $3^{e}$ période expérimentale, lorsque le suc était de nouveau réintroduit dans le duodénum. La concentration était alors de 80 et $91 \%$ de la valeur moyenne observée sur l'ensemble des périodes de restitution, soit $5,1 \pm 0,6 \mathrm{mg} / \mathrm{ml}$.

Niveaux plasmatiques des hormones.

1. Sécrétine (tabl. 1). - Dès les premières 15 min de dérivation du suc pancréatique, le niveau plasmatique de la sécrétine dans la veine porte a augmenté significativement et atteint à la $30^{\circ}$ min une valeur maximum de $152,5 \%$ de la moyenne observée en périodes de restitution du suc à l'animal. Le taux est ainsi passé de $23 \mathrm{pg} / \mathrm{ml}$ à $35 \mathrm{pg} / \mathrm{ml}$ en moyenne. On a constaté par la suite une diminution progressive des valeurs jusqu'à la $90^{\circ} \mathrm{min}$ puis une nouvelle augmentation significative à la $120^{\circ}$ min de dérivation du suc. En ce qui concerne l'évolution du taux plasmatique de cette hormone dans la circulation périphérique (veine jugulaire), elle a sensiblement été similaire à celle observée dans la veine porte. On retrouve deux augmentations significatives à la $30^{\circ}$ et à la $120^{\circ} \mathrm{min}$, les autres valeurs n'étant pas significativement différentes de la valeur moyenne observée en périodes de restitution du suc $(17,7 \pm 1,8 \mathrm{pg} / \mathrm{ml})$.

2. Cholécystokinine (tabl. 1). - La dérivation du suc pancréatique n'a entraîné aucune modification significative du taux moyen de la cholécystokinine dans la veine porte, excepté à la $30^{\mathrm{e}} \mathrm{min}$ où la valeur était de $32 \%$ supérieure au taux moyen obtenu pendant les périodes de restitution $(38,6 \pm 2,8 \mathrm{pg} / \mathrm{ml})$.

Dans la circulation périphérique, les taux moyens plasmatiques de la cholécystokinine en période de dérivation du suc n'ont pas été significativement différents du taux moyen observé en périodes de restitution $(27,6 \pm 1,7 \mathrm{pg} / \mathrm{ml})$.

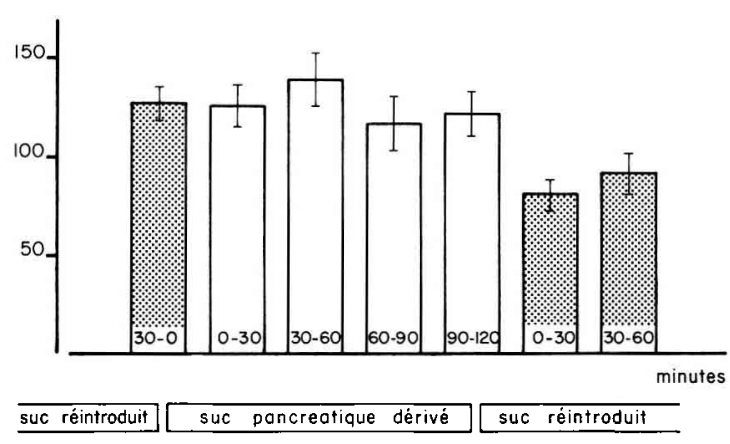

FIG. 3. - Concentration en protéines totales du suc pancréatique sécrété par $30 \mathrm{mn}$ chez le Porc pendant la période de dérivation du suc de la lumière intestinale. Valeurs exprimées en pourcentage de la moyenne des données déterminées pendant les 2 périodes de restitution du suc à I'animal. 


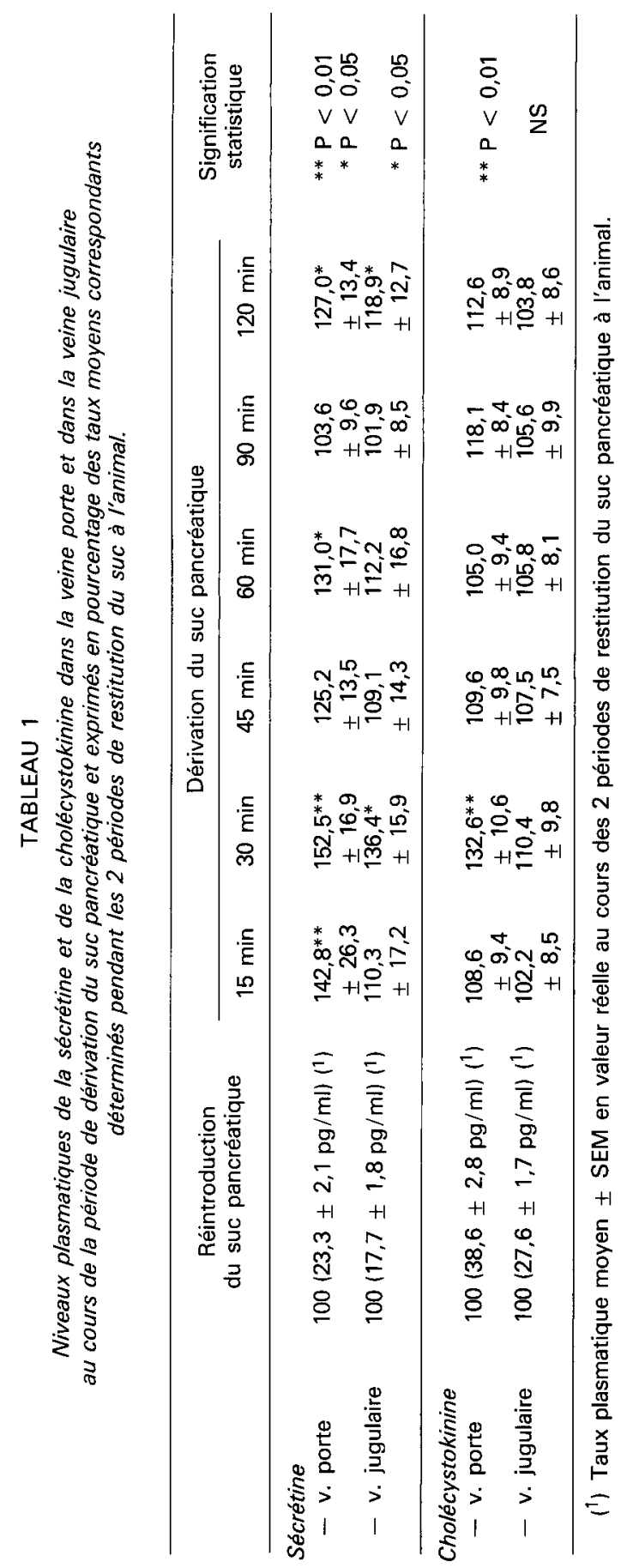


3. Gastrine, polypeptide pancréatique, VIP, somatostatine (tabl. 2). - Quelle que soit I'hormone considérée, la dérivation de la sécrétion pancréatique de l'intestin n'a entraîné aucune modification significative du taux plasmatique de ces quatre peptides dans la veine porte et dans la veine jugulaire.

\section{Discussion.}

Chez le Porc (Corring, 1974 ; Ihse et Lilja, 1979 ; Vantini et al., 1982), le Rat (Green et Lyman, 1972) ou le Hamster (Andren-Sandberg et Ihse, 1983), la dérivation du suc pancréatique hors de la lumière intestinale proximale entraîne une augmentation de la quantité de protéines sécrétées, soit une augmentation de la sécrétion enzymatique puisqu'on admet que dans le suc pancréatique la sécrétion protéique est le reflet de cette dernière. Cette observation est confirmée dans la présente étude et les résultats permettent de souligner que chez le Porc l'augmentation de la quantité de protéines pancréatiques sécrétées est en grande partie due à une forte augmentation du volume de suc écoulé. Chez le Rat, Green et Lyman (1972) observent une forte augmentation de la concentration en protéines, mais par contre un léger accroissement du volume recueilli. Nos résultats mettent de plus en évidence un effet très prononcé de la réintroduction du suc après dérivation. Le débit moyen (protéines totales et volumes) est nettement plus faible au cours de cette période que pendant la période de réintroduction initiale $(\mathrm{P}<0,05)$. II semblerait donc que l'effet de rétroaction négative lié à la présence du suc pancréatique dans la lumière duodénale soit nettement plus important que l'effet inverse, à savoir la levée de l'inhibition exercée par le suc lorsqu'il est dérivé.

Le mécanisme impliqué dans la rétroaction négative reste mal connu. Nos résultats préliminaires (Simoes-Nunes, Corring et Chayvialle, 1981) semblaient confirmer l'hypothèse selon laquelle les enzymes pancréatiques agissant sur la muqueuse duodénale influenceraient la libération d'hormones intestinales capables de moduler la sécrétion pancréatique chez le Porc (Corring, 1974) comme chez le Rat (Green et Lyman, 1972; Schneeman et Lyman, 1975) ou le Hamster (Andren-Sandberg et Ihse, 1983). La dérivation du suc pancréatique entrainerait une interruption du contrôle exercé par les enzymes, et donc soit l'accroissement du taux circulant d'hormones sécrétagogues, soit la diminution du taux circulant d'hormones inhibitrices.

L'étude présente a concerné quatre peptides proprement hormonaux (la sécrétine, la gastrine, la cholécystokinine et le PPI, le VIP à sécrétion essentiellement neuropeptidique, et la somatostatine dont le statut couramment admis est celui d'un facteur paracrine. Dans les conditions expérimentales choisies, seule la libération de sécrétine a été significative en sang systémique, confirmant nos premiers résultats (Simoes-Nunes, Corring et Chayvialle, 1981). Cette libération est survenue en dépit de la perfusion intestinale de la solution d'électrolytes à $\mathrm{pH} 8,4$, alors que l'acidification est le facteur essentiel de libération de la sécrétine chez toutes les espèces étudiées. La perfusion intestinale de la solution d'électrolytes maintient un $\mathrm{pH}$ intraduodénal entre des valeurs allant de 6,5 à 7,5 (non publié). 


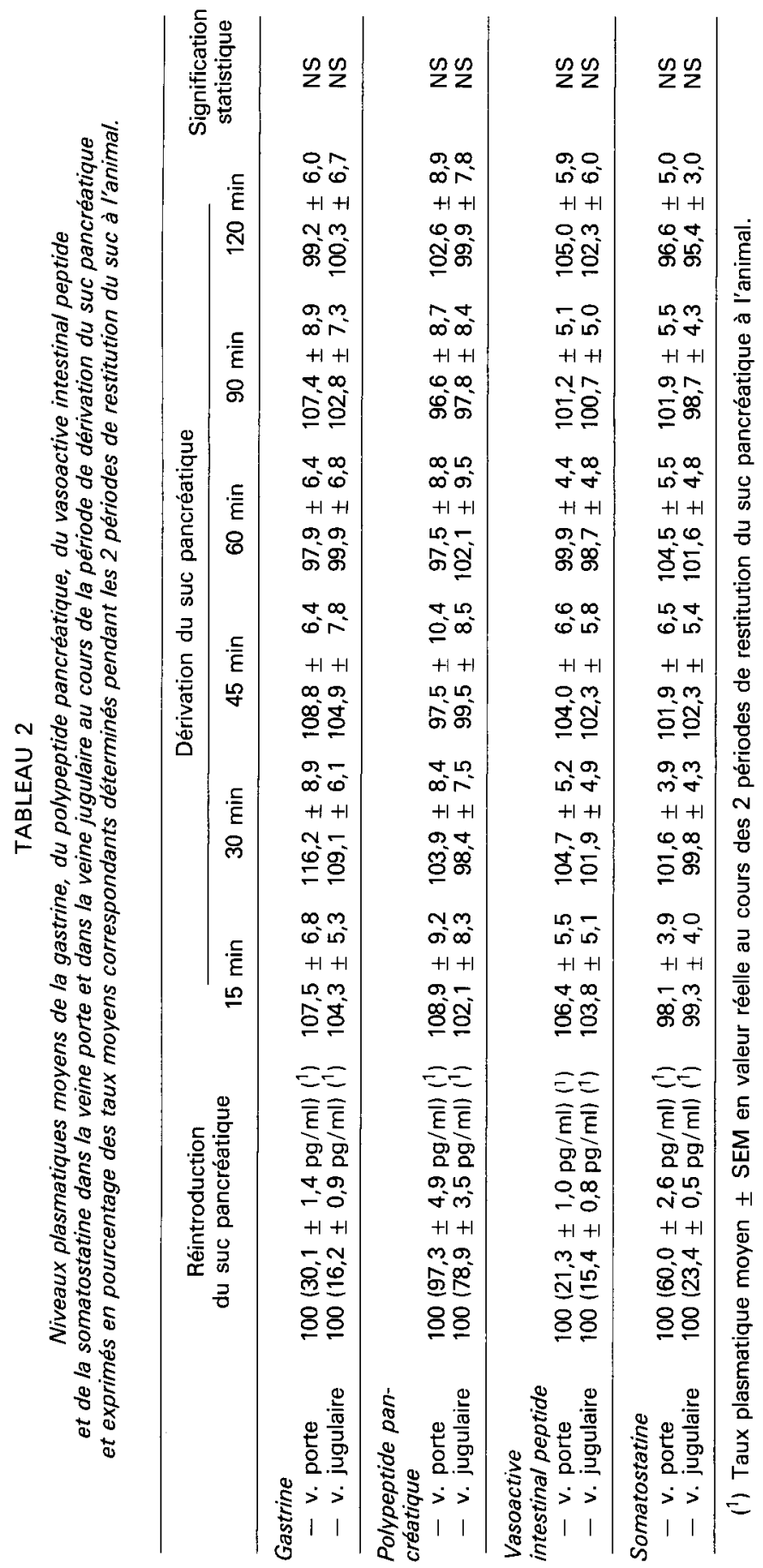


Bien que l'augmentation de la sécrétinémie ait été modérée, cette variation a probablement contribué à la réponse du pancréas exocrine. En effet, des augmentations de même ordre, induites par perfusion de peptide exogène, sont suivies d'effets significatifs chez le Porc (Schaffalitzky de Muckadell, Fahrenkrug et Holst, 1977) et chez l'Homme (Schaffalitzky de Muckadell et al., 1979). De plus, le profil de la sécrétion pancréatique et celui de la sécrétinémie au cours de la période de dérivation ont été concordants, montrant une baisse transitoire, et de mécanisme obscur, entre la $60^{\circ}$ et la $90^{\circ} \mathrm{min}$. II ne s'agit vraisemblablement pas là d'un phénomène aléatoire puisque ces variations ont été observées en même temps chez les mêmes animaux et ce chez tous les porcs. Chez le porc anesthésié (Schaffalitzky de Muckadell, Fahrenkrug et Holst, 1977), I'augmentation de la dose de sécrétine exogène perfusée par voie portale de 0,03 à 3,0 unités cliniques $\mathrm{kg}^{-1} \mathrm{~h}^{-1}$ entraîne une augmentation parallèle du débit de suc pancréatique mais une diminution de la concentration de protéines de ce dernier.

Dans l'étude rapportée ici, la dérivation du suc n'a entraîné aucune variation de la concentration protéique de la sécrétion pancréatique et aucune libération significative de CCK n'a été observée en sang périphérique. Seule une élévation transitoire était reconnue en sang porte après $30 \mathrm{~min}$ de dérivation. L'absence de variation de la concentration protéique du suc va à l'encontre de l'effet de la sécrétine seule décrit par Schaffalitzky de Muckadell, Fahrenkrug et Holst (1977) et ne nous permet pas d'éliminer la possibilité d'un effet " CCK-like ». Nos résultats doivent être interprétés à partir des caractéristiques du dosage utilisé. En effet, les variations de CCK plasmatique ont pu être sous-estimées, si la forme moléculaire prédominante dans le plasma de porc est un peptide plus petit que la nonapeptide C-terminal (Rehfeld, Holst et Jensen, 1982). Une étude approfondie des formes moléculaires circulantes doit être réalisée avant d'affirmer l'absence de libération de CCK après dérivation du suc pancréatique.

L'absence d'implication de la CCK dans les conséquences de la dérivation chez le Porc n'est pas directement transposable à d'autres espèces, en particulier au Rat. Dans cette espèce, l'effet de la rétroaction négative des enzymes pancréatiques sur la libération de CCK pourrait rendre compte de la stimulation du pancréas observée après ingestion de l'inhibiteur trypsique contenu dans le tourteau de soja cru (Green et Lyman, 1972 ; Schneeman et Lyman, 1975), puisqu'on observe dans ces conditions une élévation de la concentration plasmatique de la CCK et une hypertrophie du pancréas (Brand et Morgan, 1981). Chez le Porc, en revanche, le soja cru n'entraîne ni élévation de la CCK plasmatique, ni hypertrophie pancréatique (Yen, Jensen et Simon, 1977 ; Corring, Gueugneau et Chayvialle, à publier), I'effet de sa consommation n'est suivi que d'une augmentation du volume de sécrétion pancréatique (Schumann et al., 1983).

Les autres peptides testés ici n'ont pas varié de façon significative lors de la dérivation, malgré le contrôle en sang portal. Sans préjuger de l'intervention de mécanismes nerveux, nous pouvons suggérer que chez le Porc la fraction protéique du suc pancréatique (Corring, 1974) exerce un contrôle négatif sur cette sécrétion en inhibant la libération de sécrétine. La dérivation du suc entraîne à l'inverse une élévation de la sécrétinémie, qui intervient malgré le maintien du $\mathrm{pH}$ intraduodénal alcalin ou neutre. En ce qui concerne la $C C K$, les résultats obtenus 
ne permettent pas d'aboutir à une conclusion quant à l'implication de ce peptide dans le phénomène de rétroaction négative de la sécrétion pancréatique chez le Porc.

Reçu en juillet 1984.

Accepté en novembre 1984.

\section{References}

ANDREN-SANDBERG A., IHSE I., 1983. Regulatory effects on the pancreas of intraduodenal pancreatic juice and trypsin in the Syrian Golden hamster. Scand. J. Gastroenterol., 18, 697706.

BRAND S. J., MORGAN R .G. H., 1981. The release of rat intestinal cholecystokinin after oral trypsin inhibitor measured by bio-assay. J. Physiol., 319, 325-343.

CHAYVIALLE J. A., DESCOS F., BERNARD C., MARTIN A., BARBE C., PARTENSKY C., 1978. Somatostatin in mucosa of stomach and duodenum in gastroduodenal disease. Gastroenterology, 75, 13-19.

CHAYVIALLE J. A., MIYATA M., RAYFORD P. L., THOMPSON J. C., 1980 . Effects of test meal, intragastric nutrients, and intraduodenal bile on plasma concentrations of immunoreactive somatostatin and vasoactive intestinal peptide in dogs. Gastroenterology, 79, 844852.

CORRING T., 1974. Régulation de la sécrétion pancréatique par rétro-action négative chez le Porc. Ann. Biol. anim. Bioch. Biophys., 14, 487-498.

CORRING T., AUMAITRE A., RÉRAT A., 1972. Fistulation permanente du pancréas exocrine chez le porc. Application : réponse de la sécrétion pancréatique au repas. Ann. Biol. anim. Bioch. Biophys., 12, 109-124.

DLUGOSZ J., FÖLSCH U. R., CREUTZFELDT W., 1983. Inhibition of intraduodenal trypsin does not stimulate exocrine pancreatic secretion in man. Digestion, 26, 197-204.

FOURMY D., PRADAYROL L., ANTONIOTTI H., ESTEVE J. P., RIBET A., 1982. Purification of radio-iodinated cholecystokinin peptides by reverse phase HPLC. J. Liquid Chromat., 5, 757766.

GREEN G. M., LYMAN R. L., 1972. Feed back regulation of pancreatic enzyme secretion as a mechanism for trypsin inhibitor-induced hypersecretion in rats. Proc. Soc. exp. Biol. Med., 140, 6-12.

IHSE I., LILJA P., 1979. Effects of intestinal amylase and trypsin on pancreatic secretion in the pig. Scand J. Gastroenterol., 14, 1009-1013.

- IHSE I., LILJA P., LUNDQUIST I., 1977. Feed back regulation of pancreatic enzyme secretion by intestinal trypsin in man. Digestion, 15, 303-308.

LAPORTE J. C., 1970. Activation et sécrétion des enzymes pancréatiques. Thèse Dr. Sci., Paris.

LAPORTE J. C., TRÉMOLIĖRES J., 1973. Action de la trypsine et des inhibiteurs trypsiques sur la sécrétion pancréatique. Nutr. Métabol., 15, 192-206.

LOWRY O. H., ROSEBROUGH N. J., FARRAND A. L., RANDALL R. J., 1951. Protein measurement with the Folin-phenol reagent. J. biol. Chem., 193, 265-275.

OSNES M., HANSSEN L. E., 1980. The influence of intraduodenal administration of pancreatic juice on the bile-induced pancreatic secretion and immunoreactive secretin release in man. Scand. J. Gastroenterol., 15, 1041-1047.

PELLETIER M. J., CHAYVIALLE J. A., MINAIRE Y., 1978. Uneven and transient secretin release after a liquid test meal. Gastroenterology, 75, 1124-1132.

REHFELD J. F., HOLST J. J., JENSEN L. S., 1982. The molecular nature of vascularly released cholecystokinin from the isolated perfused porcine duodenum. Regulatory Peptides, 3, 15-28.

SALE J. K., GOLDBERG D. M., FAWCETT A. N., WORMSLEY K. G., 1977. Chronic and acute studies indicating absence of exocrine pancreatic feedback inhibition in dogs. Digestion, 15, 540-555. 
SCHAFFALITZKY de MUCKADELL O. B., FAHRENKRUG J., HOLST J. J., 1977. Plasma secretin concentration and pancreatic exocrine secretion after intravenous secretin or intraduodenal $\mathrm{HCl}$ in anaesthetized pigs. Scand. J. Gastroenterol., 12, 267-272.

SCHAFFALITZKY de MUCKADELL O. B., FAHRENKRUG J., MATZEN P., RUNE S. J., WORNING H., 1979. Physiological significance of secretin in the pancreatic bicarbonate secretion. Scand. J. Gastroenterol., 14, 85-90.

SCHNEEMAN B. O., LYMAN R. L., 1975. Factors involved in the intestinal feedback regulation of pancreatic enzyme secretion in the rat. Proc. Soc. exp. Biol. Med., 148, 897-903.

SCHUMANN B., SOUFFRANT W. B., MATKOWITZ R., GEBHARDT G., 1983. Untersuchungen zur endogenen N-Sekretion in Pankreassekret beim Schwein. Wiss. Z. K. Marx Univ., Leipzig, Math. Nat. R. 32, 6, 570-575.

SCHWARTZ T. W., REHFELD J. F., STADIL F., LARSSON L. I., CHANCE R. E., MOON N., 1976. Pancreatic polypeptide response to food in duodenal ulcer patients before and after vagotomy. Lancet, 1, 1102-1104.

SIMOES-NUNES C., CORRING T., CHAYVIALLE J. A., 1981. Mécanisme du phénomène de rétroaction négative de la sécrétion pancréatique chez le Porc: résultats préliminaires (Abstr.). Reprod. Nutr. Dévelop., 21, 809.

VANTINI I., PEDERZOLI P., MAFFEZOLI G. F., BROCCO G., CAVALLINI G., PILON T., BEBINI P., EDERLE A., PIUBELLO W., BENINI L., FERRINI S., MARCHIORI N., SCURO L. A., 1982. Presence of duodenopancreatic feedback in minipigs and possible interference from the bile. Am. J. Gastroenterol., 77, 154-157.

YEN J. T., JENSEN A. H., SIMON J., 1977. Effect of dietary raw soybean and soybean trypsin inhibitor on trypsin and chymotrypsin activities in the pancreas and in small intestinal juice of growing swine. J. Nutr., 107, 156-165. 